\title{
Molecular Cloning, Characterization and Expression Analysis of Macrohage-Colony Stimulating Factor 2 Gene from Grass Carp (Ctenopharyngodon idellus)
}

\author{
Linyong Du, Lei Qin, Shangnian Wang, Lu Yang, Kun Yang, Xinyan Wang, Hong Zhou \\ School of Life Science and Technology, University of Electronic Science and Technology of China, \\ Chengdu, China \\ Email: zhouhongzh@uestc.edu.cn
}

Received June 2013

\begin{abstract}
Macrophage colony-stimulating factor (CSF-1/M-CSF) is a key factor for the differentiation, growth and survival of monocytes/macrophages and osteoclasts. The functions of M-CSF have been well characterized in mammals. In this study, we have cloned and sequenced the cDNA of M-CSF2 in grass carp. The grass carp M-CSF2 cDNA was 1487 bp in length, containing an open reading frame of 855 bp that encodes 284 amino acids. The deduced protein of grass carp M-CSF2 possessed same domains similar to its mammalian counterparts. Multiple alignments and phylogenetic tree indicate that the grass carp M-CSF2 exhibits close evolutionary relationship with its counterparts in other teleosts. Lastly, the tissue distribution results also showed that the grass carp M-CSF2 transcript was dominantly expressed in head kidney, kidney and spleen in vivo.
\end{abstract}

Keywords: Grass Carp; M-CSF2; Macrophages; Cloning; Sequence Analysis; Tissue Distribution

\section{Introduction}

From an evolutionary standpoint, the macrophages and their functions are quite conserved in several species, and these cells have been identified in almost all multicellular organisms [1,2]. Macrophage activation is thought to play a key role in innate immune response. Moreover, macrophages also can secrete several cytokines and chemokines to regulate adaptive immune response $[3,4]$. M-CSF (M-CSF1 in teleosts) is an important growth and differentiation factor of macrophages in both of fish and mammals [1,2,5]. M-CSF has also been demonstrated to be involved in several biological processes, such as bone metabolism, inflammation and pregnancy in mammals [6-8]. All forms of M-CSF initiate their effects mediated by its receptor MCSFR, a member of the class III receptor tyrosine kinase family which encoded by the $c$-fms protooncogen [9].

Mammalian MCSF has three isoforms, a secreted glycoprotein, a secreted proteoglycan and a cell surface glycoprotein. These isoforms are generated via alternative splicing, post-translational modifications and proteolytic processing [10]. The full biological activity of M-CSF requires all isoforms, because these isoforms exert specific functions on target cells which express MCSFR at their surface and they play distinct roles in inflammation and immunity [11]. Recently, several fish
M-CSF genes have been cloned, however, the known fish M-CSF genes do not generate mammalian-equivalent splicing variants $[1,2]$. In addition, two paralogous M-CSF which may be generated by whole genome/ chromosome duplication event are identified in several teleosts [2]. In spite of these, several evidences showed the presence of functional homologous of M-CSF in several lower vertebrates. The CSF-1 domain and the important cysteine residues required for formation of the M-CSF in mammalian and teleost M-CSF are conserved [2]. Furthermore, the recombinant fish M-CSFs have been found to be biologically active like their homologs in mammals, inducing differentiation and proliferation of fish monocytes and influencing pro-inflammatory gene expression in macrophages [1,12].

The grass carp (Ctenopharyngodon idellus) is a commercially important freshwater fish and popularly cultured in China and other Asian countries. In recent years, with the rapid development of the commercial aquaculture of grass carp in China, owing to the high density breeding that causes deleterious changes in the environment and the increase of susceptibilities of fish to infections, grass carp has been exposed to serious diseases, such as hemorrhagic disease, rotted gill disease and red skin disease, causing heavy economic losses. Since M-CSF represents one of the most important mediators 
of innate immune response to eliminate microorganism infections, the studies on grass carp M-CSF would give a valuable insight into the control of diseases in aquaculture industry. In our study, we isolated grass carp MCSF2 cDNA using the homology cloning approach, performed sequence analysis and phylogeny analysis, and revealed its expression patterns in various organs.

\section{Materials and Methods}

\subsection{Animals}

One-year-old Chinese grass carp, weighting 0.5 - 0.75 kg, were purchased from Chengdu Tongwei Aquatic Science and Technology Company (Chengdu, China). Tissues for expression analysis were obtained from the freshly killed fish according to the Regulation of Animal Use in Sichuan province, China.

\subsection{Total RNA Extraction and cDNA Synthesis}

Total RNA in the organs for expression analysis was extracted by using Tripure (Roche, USA) according to the manufacturer's instructions. Five $\mu \mathrm{g}$ of total RNA was subjected to reverse transcription by using Oligo $(\mathrm{dT})_{18}$ as the primer with M-MLV reverse transcriptase (Promega, Madison, MI) and stored the cDNA at $-80^{\circ} \mathrm{C}$.

\subsection{Cloning of Grass Carp M-CSF2 cDNA}

Partial sequences of grass carp M-CSF2 was identified by homology-based PCR using other known teleost M-CSF2 sequences as references, the degenerated primers were shown in Table 1. The PCR fragments were sequenced. Based on the obtained fragment, RACE was performed with gene specific primers (Table 1) following the manufacturer's instructions of GeneRacer Kit (Life Technologies, Shanghai, China). The PCR fragments was sequenced and assembled. Finally, we validated the full-length coding sequences (CDSs) of grass carp M-CSF2 with Pfu DNA polymerase (Promega).

\subsection{Sequence Analysis and Phylogeny Analysis}

The open reading frames and deduced protein sequences of grass carp M-CSF2 was identified by ORF finder [13]. The signal peptide was predicted using the SignalP 4.1 program and the transmembrane domain was predicted using the TMpred program [14,15]. Characteristic domains of the deduced proteins were predicted by SMART program [16]. The identity analysis by using nucleotide and protein BLAST programs

(www.ncbi.nlm.nih.gov/BLAST/). Multiple alignments between the deduced amino acid sequence of grass carp M-CSF2 and those of other known species M-CSF was conducted with DNAMAN software (Lynnon Biosoft,
Table 1. Primers used for gene cloning and expression analysis.

\begin{tabular}{lll}
\hline Name & Sequence (5'to 3') & objects \\
\hline M-CSF2 F & CCAAGGGGAAGAATGTGTGTG & cloning \\
M-CSF2 R & GGGAGTAGATGTTGAGGATGAG & \\
M-CSF2 5N1 & CCATGCTCAGGTGTTTTCTCTC & 5'-RACE \\
M-CSF2 5N2 & CATCATGGAAAGGGGAACACACAG & \\
M-CSF2 3N1 & GAGAGAAAACACCTGAGCATGG & 3'-RACE \\
M-CSF2 3N2 & GCTTGAACTGTTTAACGTCCACTTC & \\
M-CSF2 & CCTTGCAAACATGCCATAACCAT & MCSF2 \\
Realtime F & & expression \\
M-CSF2 & CCATGCTCAGGTGTTTTCTCT & \\
Realtime R & & \\
B-actin QF & AGCCATCCTTCTTGGGTATG & $\beta$-actin \\
B-actin QR & GGTGGGGCGATGATCTTGAT & expression \\
\hline
\end{tabular}

Quebec, Canada). Phylogenetic tree was constructed by using MEGA 4 (www.megasoftware.net) based on Neighbor-Joining method with the bootstrapping of 1000 repetitions [17]. The 3D structure model of CSF-1 domain of grass carp M-CSF2 was performed using the SWISSMODEL program (http:// swissmodel.expasy.org).

\subsection{Expression Patterns of M-CSF in Vivo}

Total RNA was extracted from the thymus, head kidney, liver, spleen, gill, intestine and muscle, the cDNAs were prepared by reverse transcription as described previously. The gene-specific primers for grass carp M-CSF2 were designed (Table 1) and used for detecting its transcript in different tissues by real-time quantitative PCR (qPCR). In our study, qPCR was performed on the Bio-Rad CFX96 Real-time detection system (Bio-Rad Laboratories, Inc., Hercules, CA) in a final volume of $20 \mu \mathrm{l}$ with $10 \mu \mathrm{l}$ iQTMSYBRTM Green Supermix (Bio-Rad Labora tories, Inc., Hercules, CA) following the procedures in our previous study. $\beta$-actin was used as a internal control. Data were analyzed using the CFX manager and normalized to $\beta$-actin after correcting for differences in amplification efficiency.

\section{Results}

\subsection{Molecular Cloning Grass Carp M-CSF2 cDNA}

The complete cDNA and deduced amino acid sequences of grass carp M-CSF2 (Genebank ID: KF113858) are shown in Figure 1. The grass carp M-CSF2 cDNA sequence is 1487 bp in length with a 216 bp 5'-untranslated region (UTR), a 416 bp 3'-UTR with the latter containing a cytokine RNA instability motif (ATTTA) and a polyadenylation signal (AATAAA) nucleotide up- 


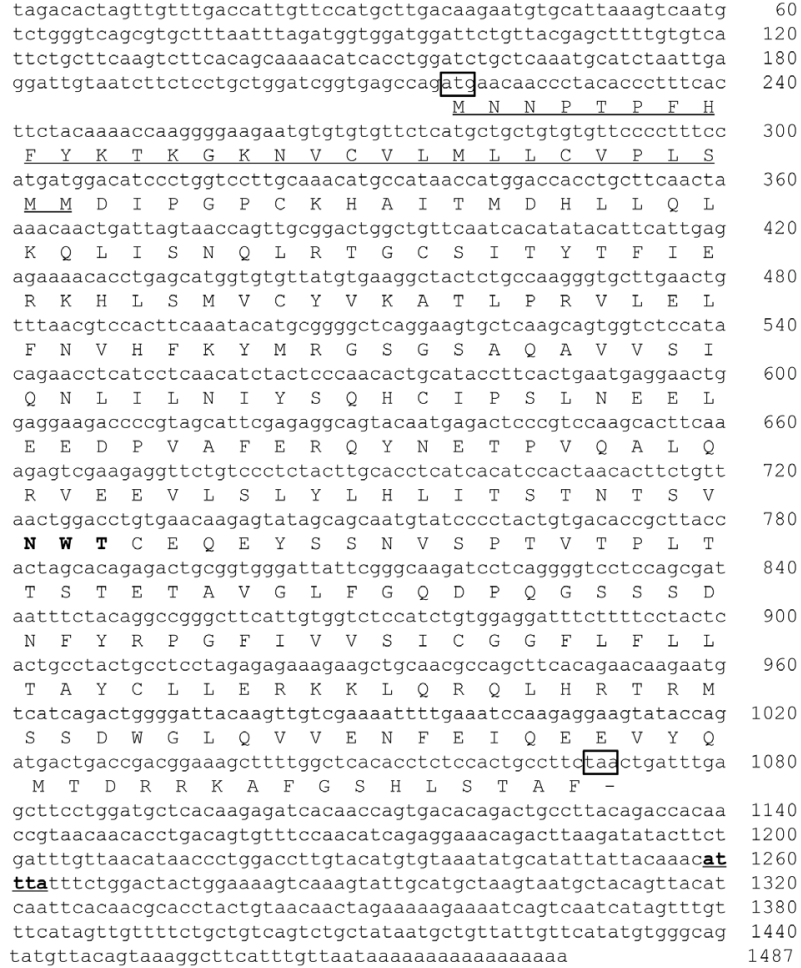

Figure 1. The cDNA sequences and deduced amino acids of grass carp M-CFS2. The translation start codon and stop codon are boxed. The signal peptide is underlined. The potential N-glycosylation sites are in bold. Within 3'UTR the putative ATTTA instability motifs are underlined and in bold.

stream of the poly (A) tail. Grass carp M-CSF2 has an 855 bp open reading frame (ORF) encoding a 284-amino acid polypeptide.

The putative protein of M-CSF2 contained one $\mathrm{N}$ glycosylation sites, and its predicted molecular weight is $32.2 \mathrm{kDa}$ and theoretical isoelectric point is 6.17 (data not shown). The putative protein of grass carp M-CSF2 showed $88.89 \%$ and $53.31 \%$ identities to its homologs in zebrafish and rainbow trout, respectively (Table 2). When compared with M-CSFs in other species, grass carp M-CSF2 shared 23.51\% - 50.63\% identity with them (Table 2). In addition, alignment of protein sequence of grass carp M-CSF2 with other species M-CSF revealed that they harbored an area of strong amino acid conservation encoding the CFS-1 domain (Figure 2, 4A). Phylogenetic tree revealed that grass carp M-CSF2 was close to the counterparts in fish and far from those of the mammalian (Figure 3). Despite the difference in size of the translated proteins and lower homology between fish and mammalian M-CSF, analysis of M-CSF using the SMART program indicated that it has a similar modular structure with other species M-CSF, containing a signal peptide, a CSF-1 domain, a transmembrane domain and an intracellular region (Figure 4(a)). To further understand the spatial structure of teleost M-CSF2, the 3D homology modeling of the grass carp M-CSF was performed by using the human M-CSF (PDB ID: 1hmc) as a template. Figure 4(b) showed that grass carp M-CSF consisted 2 parallel $\beta$-shells and 5 a-helixes that formed a global fold, which was similar to the structure of the other mammalian M-CSF.

\subsection{Tissue Distribution of Grass Carp M-CSF2}

The constitutive expression of grass carp M-CSF2 was detected in all of selected tissues. As shown in Figure 5, M-CSF mRNA was detected at the highest level in spleen, at lesser extent in kidney, head kidney and thymus, while low levels in liver, intestine and gill. Similar basal expression pattern was observed in other fishes [2].

\section{Discussion}

M-CSF is a main factor for macrophage differentiation and survival. Monocyte/Marophage is a major subset of phagocytes in vertebrates which plays a vital role in innate immune response [18]. The innate immune system is supreme important for fish in defending infections and maintaining homeostasis of the host immune system. In order to better understand teleost innate immune system and macrophage, we described the identification of grass carp M-CSF2 molecule for the first time. In addition, the M-CSF2 functional domain and 3D structural model was

Tabel 2. CDS/amino acid identity of grass carp M-CSF2 with its homologs in other vertebrates (upper triangle = anino acid identity; lower triangle $=$ CDS identity).

\begin{tabular}{|c|c|c|c|c|c|c|c|}
\hline DNA/AA & 1 & 2 & 3 & 4 & 5 & 6 & 7 \\
\hline 1. Grass carp M-CSF2 & & 27.64 & 88.89 & 23.51 & 53.31 & 50.63 & 39.29 \\
\hline 2. Zebrafish M-CSF1 & 18.27 & & 27.66 & 47.06 & 28.28 & 25.41 & 19.74 \\
\hline 3. Zebrafish M-CSF2 & 80.79 & 22.16 & & 23.42 & 53.25 & 51.20 & 39.95 \\
\hline 4. Rainbow trout M-CSF1 & 16.09 & 32.72 & 16.47 & & 25.68 & 23.67 & 18.67 \\
\hline 5. Rainbow trout M-CSF2 & 37.94 & 20.79 & 41.84 & 17.56 & & 62.56 & 42.25 \\
\hline 6. Takifugu M-CSF2 & 39.74 & 19.21 & 41.78 & 18.23 & 55.56 & & 41.95 \\
\hline 7. Human M-CSF & 19.21 & 10.15 & 21.88 & 9.02 & 18.02 & 19.05 & \\
\hline
\end{tabular}




\begin{tabular}{|c|c|c|}
\hline Gc M-CSF2 & GKNVCVLM CVPLSMMI PGP K A TMD & 47 \\
\hline Zf M-SCF2 & VKNVCVLM CVPLSMMI PGP K A TMD & 47 \\
\hline Rt M-CSF2 & TLLVPTHI QCKT. . KVKCLCVMNF SLPLTMGD. PGP R S TRD & 47 \\
\hline Tf M-CSF2 & TI LGSTLI QSKAKLQVKCLCVLMF SFPLI TTEI PGP R S TKE & 50 \\
\hline Hm M-CSF & TAPGAAGRCPPTTWLGSLLLLVCL ASRSI TEEVSEY S N GSG & 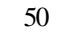 \\
\hline Consensus & c h i hl & \\
\hline M-CSF2 & YT I ERKH S. MN YV ATLPRVLELFNVHFKYM & 96 \\
\hline Zf M-SCF2 & YT I ERKH S. WV YV AALPRVLELVNVHFRYV & 66 \\
\hline Rt M-CSF2 & YT I EQRS N. KC YV AAMPW LELLTGHFRYT & 96 \\
\hline Tf M-CSF2 & YT I ERQS S. KC FV AALPW LELLTTHFKYT & 99 \\
\hline Hm M-CSF & FE VDQEQ KDPV YL KAFLLVQYI MEDTMRFR & 100 \\
\hline Consensus & l $\quad \mathrm{q} \quad \mathrm{c} \mathrm{it}$ & \\
\hline CSF2 & RGSGSAQAVVI QN I LNI Y QH I PSLNEEL EDPVAFERQYNET VQA & 46 \\
\hline $\mathrm{CF} 2$ & RGSGSAQSSWSLQ̀N I LNI Y Q̈H VPSLNEEL EDPVAFETQYYKES VQ̀A & 146 \\
\hline SF2 & RGSDNHSYVLSLTG I HNI Y QR VPQI NEEL DDPVSFGMSFSSS SEA & 14 \\
\hline CSF2 & RGSVNDGYVQSLRA I LNI Y QKK VPQ் NEEI DKPESFEMYYSGS LEA & 14 \\
\hline CSF & FT. . . KDY EHDKACVRTFYET LQL & 14 \\
\hline Consensus & $\mathrm{p}$ & \\
\hline CSF2 & QR EE LSLYLH I TSTNTSVNMT EQEYSS. NVSPTVTPLTT TETAV & \\
\hline CF2 & QR EE LSLYLH I TSI NTPVNMT EQEYSS. SDPPTVTQLTT TEAAV & \\
\hline CSF2 & GR QD LSVYWE VTTMDSPVDUS EREYTDTAEPPTALPTAL LTTVS & 15 \\
\hline CSF2 & QG SE LSLYWE VTMSKVPLDWR QQEYTEPFSSTPELPTES TQFFT & 19 \\
\hline $\mathrm{CSF}$ & EK KN FNETKN LDKDWNI FSKN NNSFAECSSQGHERQSEG & \\
\hline Consensus & $\mathrm{l} \quad \mathrm{V} \quad \mathrm{V}$ & \\
\hline Gc $N$ & SDNFYRPGFI WS. . I C GFLFLLTAYCLLERK & \\
\hline $\mathrm{CF} 2$ & GADFYRLGFI VVT. . I C GFLVLLTAYCLLERK & 23 \\
\hline SS2 & TLWDSEKAPQTSLDGDQDRDLYKFGFMM APSVC GLLFI FTLYCLI THK & 2 \\
\hline CSF2 & ESYSRESAKAS. . HRRPVRDLYKLGFI I AS. . I C GLLLI VTLYCLI AKK & 24 \\
\hline Hm M-CSF & . . . SSPQLQES. . . . . . VFHLLVPSVI LVLLAVG LLFYRWRRRSHQEPQ & 23 \\
\hline Consensus & g & \\
\hline CSF2 & KLQRQLHRTRMS. . SDWGLQVVENF I QEEVYQMIDRRKAFGSHLSTAF & 28 \\
\hline $\mathrm{CF} 2$ & RLQRQLLDRTRKS. . SDWGLQVVENY I QEEEVYQMTDRRKQFDVHLSTAF & \\
\hline Rt M-CSF2 & MSYSTDHHTSLGYQNSSLHTDI QDI MQVE. & \\
\hline Tf M-CSF2 & KI RSPHKSKSS. . . TNSSRDLEDI MEPQ. & 27 \\
\hline Hm M-CSF & RADSPLEQPEG. . . . SPLTQDDRQV LPV. . & \\
\hline Conse & & \\
\hline
\end{tabular}

Figure 2. Multiple alignment of the predicted grass carp M-CSF2 deduced protein sequences with MCSFs in other species. The conserved amino acid residues were highlighted by shadows. GcM-CSF2, grass carp M-CSF2; Zf M-CSF2, zebrafish M-CSF2; Rt M-CSF2, rainbow trout M-CSF2; Tf M-CSF2, takifugu M-CSF2; Hm M-CSF, human M-CSF.

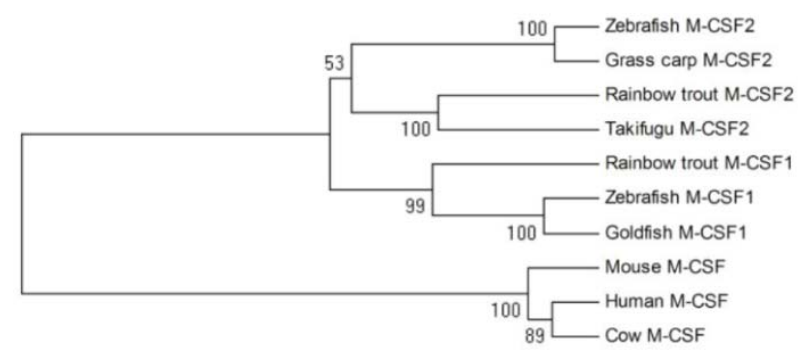

Figure 3. Phylogenetic tree of M-CSF amino acid sequences was constructed with the neighbor-joining algorithm by using MEGA4 program. The branches were validated by bootstrap analysis from 1000 replications, which are represented by percentage in branch nodes. The GeneBank accession numbers used in this study, zebrafish M-CSF2, AM901599; zebrafish M-CSF1, AM901598; rainbow trout M-CSF2, AM949839; rainbow trout M-CSF1, AJ555867; goldfish M-CSF1, AM982798; takifuguM-CSF2, BAM75189; mouse M-CSF, P07141; human M-CSF, P09603; Cow M-CSF, O77709.

analyzed using bioinformatics.

The grass carp M-CSF2 ORF was 855bp and showed a relatively high degree of nucleotide sequence identity with the three known fish M-CSF2 cDNAs, whereas, when compared with fish M-CSF1 and mammalian M-CSF, it exhibited a low identities with those genes. In 3'-UTR of grass carp M-CSF2, one cytokine RNA instability motif (ATTTA), which are typical cytokine genes, and a polyadenylation signal (AATAAA) were found, indicating that the M-CSF2 mRNA expression was tightly regulated $[19,20]$.

The putative protein of grass carp M-CSF2 shared similar molecular weight with other fish homologs. Despite that grass carp M-CSF exhibited low identity with human M-CSF, both fish M-CSFs and human M-CSF share similar structure: a signal peptide, a CSF-1 domain, a transmembrane domain and an intracellular domain. Furthermore, grass carp M-CSF2 share relatively higher homology with human M-CSF at CSF-1 domain, which is important for M-CSF function in mammals [2]. In addition, the alignment result of M-CSFs revealed that grass carp M-CSF2 are highly conserved with zebrafish homologs. Meanwhile, zebrafish M-CSF2 gene is conserved in syntenic chromosomal relationships compared with human M-CSF [2]. Moreover, high similarity of 3D models between human M-CSF and grass carp M-CSF2 was observed. All evidence described above indicates 


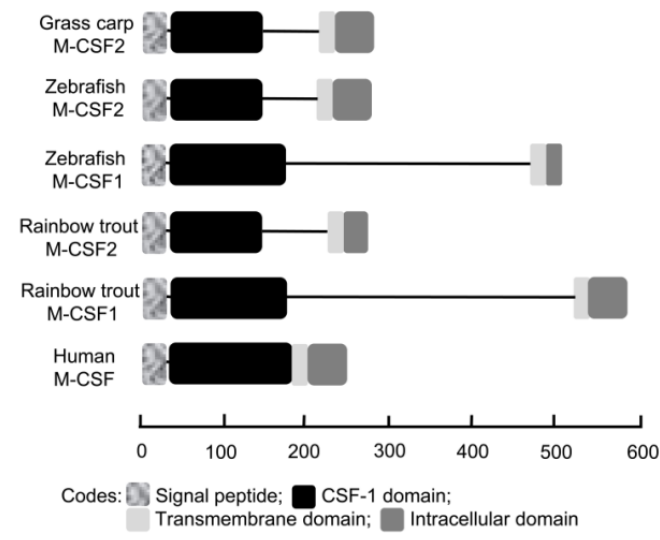

(a)

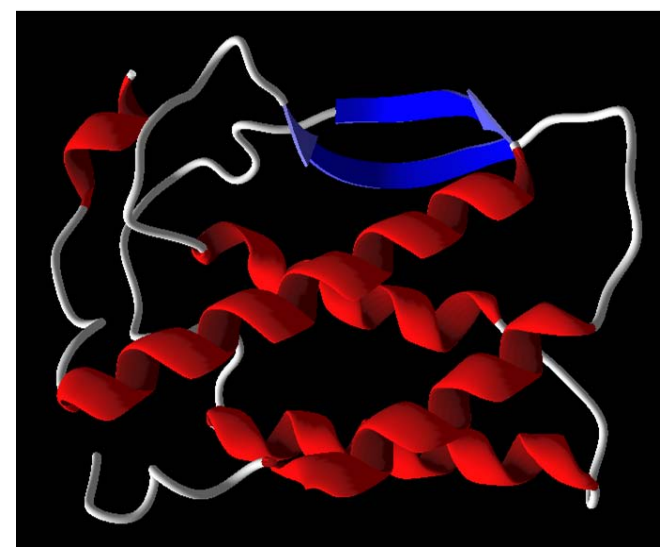

(b)

Figure 4. (a) Domains of M-CSFs molecule. All M-CSFs have signal peptide, CSF-1 domain, transmembrane domain and intracellular region; (b) 3D structure of CSF-1 domain in grass carp M-CSF2. Backbone ribbon and the secondary structure topology are shown: blue arrows represent beta strands, red cylinders represent alpha helixes.

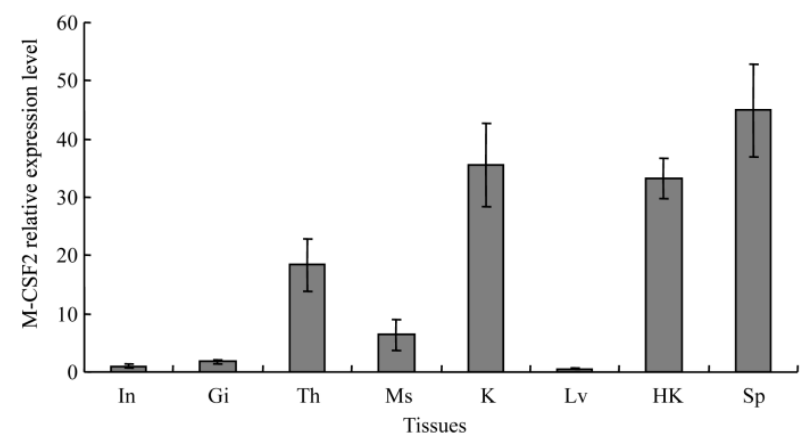

Figure 5. Expression pattern of grass carp M-CSF2 in different tissues. qPCRs were performed using primers specific for IL-1 $\beta$ and $\beta$-actin. $\beta$-actin was amplified as internal control, and then expressed as the ratio of the mRNA level In. In, intestine; Gi, gill; T, thymus; Ms, musle; K, kidney; L, liver; HK, head kidney; Sp, spleen.

that the newly cloned grass carp M-CSF2 is indeed the ortholog of mammal M-CSF in grass carp.
In mammals, M-CSF is known as an important inflammatory cytokine, in agreement with immunological function in mammals, grass carp M-CSF2 transcript was dominantly expressed in head kidney, spleen, kidney and thymus. Actually, all of these tissues are considered as the major lymphoid organs in teleosts.

In summary, our data showed that grass carp M-CSF2 was structurally similar to its counterpart in mammals and exhibited relatively higher expression level in immune-related tissues, providing the basis for further investigation of its immune role in grass carp.

\section{Acknowledgements}

This work was supported by grant from the National Natural Science Foundation of China (31101877).

\section{REFERENCES}

[1] P. C. Hanington, et al., "Growth Factors of Lower Vertebrates: Characterization of Goldfish (Carassius auratus L.) Macrophage Colony-Stimulating Factor-1,” The Journal of Biological Chemistry, Vol. 282, 2007, pp. 31865-31872. http://dx.doi.org/10.1074/jbc.M706278200

[2] T. Wang, et al., "Two Macrophage Colony-Stimulating Factor Genes Exist in Fish That Differ in Gene Organization and Are Differentially Expressed," The Journal of Immunology, Vol. 181, 2008, pp. 3310-3322.

[3] C. A. Janeway Jr. and R. Medzhitov, "Innate Immune Recognition,” Annual Review of Immunology, Vol. 20, 2002, pp. 197-216.

http://dx.doi.org/10.1146/annurev.immunol.20.083001.08 $\underline{4359}$

[4] F. O. Martinez, et al., "Alternative Activation of Macrophages: An Immunologic Functional Perspective,” Annual Review of Immunology, Vol. 27, 2009, pp. 451-483. http://dx.doi.org/10.1146/annurev.immunol.021908.1325 $\underline{32}$

[5] V. Chitu and E. R. Stanley, "Colony-Stimulating Factor-1 in Immunity and Inflammation," Current Opinion in Immunology, Vol. 18, 2006, pp. 39-48.

http://dx.doi.org/10.1016/j.coi.2005.11.006

[6] P. E. Cohen, et al., "Absence of Colony-Stimulating Factor-1 in Osteopetrotic (Csfmop/Csfmop) Mice Results in Male Fertility Defects,” Biology of Reproduction, Vol. 55, 1996, pp. 310-317. http://dx.doi.org/10.1095/biolreprod55.2.310

[7] P. E. Cohen, et al., "Absence of Colony Stimulating Factor-1 in Osteopetrotic (Csfmop/Csfmop) Mice Disrupts Estrous Cycles and Ovulation,” Biology of Reproduction, Vol. 56, 1997, pp. 110-118. http://dx.doi.org/10.1095/biolreprod56.1.110

[8] L. R. Rohrschneider, et al., "Growth and Differentiation Signals Regulated by the M-CSF Receptor,” Molecular Reproduction and Development, Vol. 46, 1997, pp. 96103.

http://dx.doi.org/10.1002/(SICI)1098-2795(199701)46:1< 96::AID-MRD15>3.0.CO;2-1 
[9] X. M. Dai, et al., “Targeted Disruption of the Mouse Colony-Stimulating Factor 1 Receptor Gene Results in Osteopetrosis, Mononuclear Phagocyte Deficiency, Increased Primitive Progenitor Cell Frequencies, and Reproductive Defects,” Blood, Vol. 99, 2002, pp. 111-120. http://dx.doi.org/10.1182/blood.V99.1.111

[10] P. Fixe and V. Praloran, "M-CSF: Haematopoietic Growth Factor or Inflammatory Cytokine?” Cytokine, Vol. 10, 1998, pp. 32-37. http://dx.doi.org/10.1006/cyto.1997.0249

[11] M. J. Sweet and D. A. Hume, "CSF-1 as a Regulator of Macrophage Activation and Immune Responses," Archivum Immunologiae et Therapia Experimentalis (Warsz), Vol. 51, 2003, pp. 169-177.

[12] L. Grayfer, et al., "Macrophage Colony-Stimulating Factor (CSF-1) Induces Pro-Inflammatory Gene Expression and Enhances Antimicrobial Responses of Goldfish (Carassius auratus L.) Macrophages," Fish and Shellfish Immunology, Vol. 26, 2009, pp. 406-413. http://dx.doi.org/10.1016/j.fsi.2008.12.001

[13] I. T. Rombel, et al., "ORF-FINDER: A Vector for HighThroughput Gene Identification,” Gene, Vol. 282, 2002, pp. 33-41. http://dx.doi.org/10.1016/S0378-1119(01)00819-8

[14] J. D. Bendtsen, et al., "Improved Prediction of Signal Peptides: SignalP 3.0,” Journal of Molecular Biology, Vol. 340, 2004, pp. 783-795. http://dx.doi.org/10.1016/S0378-1119(01)00819-8
[15] M. Ikeda, et al., “Transmembrane Topology Prediction Methods: A Re-Assessment and Improvement by a Consensus Method Using a Dataset of Experimentally-Characterized Transmembrane Topologies,” In Silico Biology, Vol. 2, 2002, pp. 19-33.

[16] J. Schultz, et al., "SMART, a Simple Modular Architecture Research Tool: Identification of Signaling Domains," Proceedings of the National Academy of Sciences of the United States of America, Vol. 95, 1997, pp. 5857-5864. http://dx.doi.org/10.1073/pnas.95.11.5857

[17] K. Tamura, et al., "MEGA4: Molecular Evolutionary Genetics Analysis (MEGA) Software Version 4.0,” Molecular Biology and Evolution, Vol. 24, 2007, pp. 15961599. http://dx.doi.org/10.1093/molbev/msm092

[18] D. A. Hume, et al., "The Mononuclear Phagocyte System Revisited," Journal of Leukocyte Biology, Vol. 72, pp. 621-627.

[19] A. Bonnieu, et al., “AUUUA Motifs Are Dispensable for Rapid Degradation of the Mouse c-myc RNA," Oncogene, Vol. 5, 1990, pp. 1585-1588.

[20] S. Savant-Bhonsale and D. W. Cleveland, "Evidence for Instability of mRNAs Containing AUUUA Motifs Mediated through Translation-Dependent Assembly of a > 20S Degradation Complex,” Genes \& Development, Vol. 6, 1992, pp. 1927-1939. http://dx.doi.org/10.1101/gad.6.10.1927 